\title{
特殊形貌磷酸锆钠粉体的制备与表征
}

\author{
陈军军，王晶，惠香
}

(大连交通大学 辽宁省无机超细粉体制备及应用重点实验室，大连 116028)

摘 要: 采用水热法、以八水合氧氯化锆和多聚磷酸钠为原料、水为介质, 一步制备出了单相 $\mathrm{Na}_{2} \mathrm{Zr}\left(\mathrm{PO}_{4}\right)_{2}$ 粉体。利 用 XRD、SEM、TG-DSC 等检测方法, 系统地考察了水热条件和㷽烧温度对磷酸铅钠粉体微观结构的影响。结果 表明, 原料摩尔配比为 $n\left(\mathrm{ZrOCl}_{2} \cdot 8 \mathrm{H}_{2} \mathrm{O}\right): n\left(\mathrm{Na}_{5} \mathrm{P}_{3} \mathrm{O}_{10}\right)=1: 2$ 、无需调变 $\mathrm{pH}$ 值的中性条件下, 于 $140^{\circ} \mathrm{C}$ 水热 $14 \mathrm{~h}$, 即可 制得分散性较好、尺寸较均一、由片组装而成的类线团形貌的 $\mathrm{Na}_{2} \mathrm{Zr}\left(\mathrm{PO}_{4}\right)_{2}$ 粉体; 适当延长水热时间和提高水热温 度均有利于粉体的形成，且温度越高，形成该粉体所需水热时间越短。高温㷽烧会使 $\mathrm{Na}_{2} \mathrm{Zr}\left(\mathrm{PO}_{4}\right)_{2}$ 粉体发生热分解， 其形貌也被破坏。利用生长基元模型解释了水热法生成该磷酸铅钠粉体的机理。

关 键 词: $\mathrm{Na}_{2} \mathrm{Zr}\left(\mathrm{PO}_{4}\right)_{2}$; 特殊形貌; 水热法; 生长基元; 怖烧

中图分类号: TQ126 文献标识码: A

\section{Preparation and Characterization of Sodium Zirconium Phosphate Powder with Peculiar Morphology}

\author{
CHEN Jun-Jun, WANG Jing, HUI Xiang
}

\begin{abstract}
(Liaoning Key Laboratory for Fabrication and Application of Superfine Inorganic Powders, Dalian Jiaotong University, Dalian 116028, China)
\end{abstract}

\begin{abstract}
Single-phase $\mathrm{Na}_{2} \mathrm{Zr}\left(\mathrm{PO}_{4}\right)_{2}$ powders were directly synthesized by hydrothermal method using $\mathrm{ZrOCl}_{2} \cdot 8 \mathrm{H}_{2} \mathrm{O}$ and sodium polyphosphate as raw materials, water as solvent. The effects of hydrothermal conditions and calcination temperature on the microstructure of sodium zirconium phosphate powders were investigated systemically by SEM, $\mathrm{XRD}$ and TG-DSC, etc. It is found that, when the molar ratio of raw materials is 1:2 $\left(n\left(\mathrm{ZrOCl}_{2} \cdot 8 \mathrm{H}_{2} \mathrm{O}\right): n\left(\mathrm{Na}_{5} \mathrm{P}_{3} \mathrm{O}_{10}\right)\right)$ without adjusting $\mathrm{pH}$ value, slices being assembled coil-like $\mathrm{Na}_{2} \mathrm{Zr}\left(\mathrm{PO}_{4}\right)_{2}$ powders with good dispersion and uniform size can be obtained after $14 \mathrm{~h}$ hydrothermal treatment at $140^{\circ} \mathrm{C}$. Appropriate extension of time and increase of temperature are beneficial to the powder formation. The higher the temperature, the shorter time the desired powders form. After calcination, $\mathrm{Na}_{2} \mathrm{Zr}\left(\mathrm{PO}_{4}\right)_{2}$ powders decompose and their morphology are destroyed. The growth mechanism of the sodium zirconium phosphate powders being prepared by hydrothermal method is explained by a basic growth unit model.
\end{abstract}

Key words: $\mathrm{Na}_{2} \mathrm{Zr}\left(\mathrm{PO}_{4}\right)_{2}$; peculiar morphology; hydrothermal method; growth unit; calcination

磷酸锆钠材料由于其良好的快离子导电性、低 的热膨胀系数、低的导热系数和作为催化剂载体的 耐热冲击性，被广泛应用于航空航天、汽车工业、 催化剂载体、军事领域的高温导弹、小型换热器、
核废料处理等方面 ${ }^{[1-3]}$, 另外材料的微观形貌与其 性能有着密切的联系 ${ }^{[4-5]}$, 所以特殊形貌的磷酸锆 钠可能拥有更优良的性能，从而提高其利用价值。 目前，国内外生产磷酸锆钠粉体主要采用高温固相 
法和沉淀法。但通过这两种方法制得的粉体纯度、 粒径、形貌都难以控制, 且产物的分散性不好。此 外沉淀法还会造成大量的磷酸浪费, 大大限制了磷 酸锆钠材料的生产及应用 ${ }^{[1,6-7]}$ 。为了解决上述问题, 本研究以多聚磷酸钠作为磷酸锆钠中磷酸钠的来源, 采用水热法制备粉体，目的是制备出粒度均匀、形 貌可控的磷酸锆钠粉体 ${ }^{[8-10]}$, 同时为了确定这种磷 酸锆钠的可用温度范围, 考察了煅烧温度对其晶形 及形貌的影响。此外, 透彻理解溶液中的晶体生长 机理对制备工艺的改进、理想结构和性能的获得至 关重要。本研究采用生长基元模型解释了这种磷酸 锆钠粉体的生长过程 ${ }^{[1-14]}$ 。

\section{1 实验方法}

\section{1 原料}

实验所采用的八水合氧氯化锆 $\left(\mathrm{ZrOCl}_{2} \cdot 8 \mathrm{H}_{2} \mathrm{O}\right)$ 购于国药集团化学试剂有限公司; 多聚磷酸钠(以 $\mathrm{Na}_{5} \mathrm{P}_{3} \mathrm{O}_{10}$ 计)购于天津市大茂化学试剂厂; 氢氧化钠 购于天津市大陆化学试剂厂; 盐酸购于新光化工试剂 厂; 所有试剂均为分析纯。去离子水为实验室自制。

\section{2 样品制备}

将 $3.3 \mathrm{~g}$ 多聚磷酸钠和不同质量的八水合氧氯化 锆加入到 $75 \mathrm{~mL}$ 水中, 搅拌均匀后倒入容积为 $100 \mathrm{~mL}$ 带聚四氟乙烯内祄的不锈钢高压釜中，在不同的水 热条件下处理, 处理后的样品用去离子水清洗数次 后，放入 $60^{\circ} \mathrm{C}$ 烘箱内干燥，制得粉体样品。再将部 分粉体样品进行炦烧处理。

\section{3 样品表征}

利用荷兰帕纳科公司生产的 Empyrean $\mathrm{X}$ 射线 衍射仪对产物的晶相结构进行表征 $(\mathrm{Cu} \mathrm{K} \alpha$ 辐射, $\lambda=$ $0.15418 \mathrm{~nm}$ )。通过扫描电子显微镜(日本 JEOL 公司 JSM-6360LV 型)观察产物的形貌和粒径。采用德国 耐驰 STA449F3 同步热分析仪测定样品在升温速率 为 $10 \mathrm{~K} / \mathrm{min}$, 从室温升温到 $900^{\circ} \mathrm{C}$ 条件下的 TG-DSC 曲线。

\section{2 结果与讨论}

\section{1 原料配比及 $\mathrm{pH}$ 值的影响}

图 1 给出了加入不同摩尔比的 $\mathrm{ZrOCl}_{2} \cdot 8 \mathrm{H}_{2} \mathrm{O}$ 和 $\mathrm{Na}_{5} \mathrm{P}_{3} \mathrm{O}_{10}$ 在 $140^{\circ} \mathrm{C}$ 下反应 $12 \mathrm{~h}$ 获得产物的 XRD 图 谱。由图 1 可知, 当摩尔比为 1:1.2 和 1:1.4 时, XRD 图谱没有明显的衍射峰, 当摩尔比为 $1: 1.6 、 1: 1.8$ 、 1:2.0、1:2.4 时, 产物有相似的 XRD 图谱, 各峰与 $\mathrm{Na}_{2} \mathrm{Zr}\left(\mathrm{PO}_{4}\right)_{2}$ 的标准峰值(JCPDS 24-1178) 一致, 可知
晶相结构均为 $\mathrm{Na}_{2} \mathrm{Zr}\left(\mathrm{PO}_{4}\right)_{2}$, 另外当摩尔比为 $1: 1.8$ 和 1:2.0 时, 产物的峰强较强, 峰形也较窄, 说明此 时产物晶相发育相对较好。

图 2 给出了加入不同摩尔比的 $\mathrm{ZrOCl}_{2} \cdot 8 \mathrm{H}_{2} \mathrm{O}$ 和 $\mathrm{Na}_{5} \mathrm{P}_{3} \mathrm{O}_{10}$ 在 $140^{\circ} \mathrm{C}$ 下反应 $12 \mathrm{~h}$ 获得产物的 SEM 照 片。由图 2 可以看出, 当摩尔比为 1:1.8、1:2.0 和 1:2.4 时, 获得的产物均出现了六角片插组装成的类 线团结构, 但摩尔比为 1:2.0 时得到的产物分散性较 好, 形貌较一致, 粒径较为均一。而当摩尔比为 1:1.6 时形成的产物开始解理为六角片状结构。

表 1 为加入不同摩尔比的 $\mathrm{ZrOCl}_{2} \cdot 8 \mathrm{H}_{2} \mathrm{O}$ 和 $\mathrm{Na}_{5} \mathrm{P}_{3} \mathrm{O}_{10}$ 水热前后溶液的 $\mathrm{pH}$ 值。由表 1 可知, 配比的改变, 会轻微改变初配液的 $\mathrm{pH}$ 值, 当摩尔比为 $1: 2.0$ 时恰 使初配溶液呈中性。而水热反应后溶液的 $\mathrm{pH}$ 值均 呈弱酸性(水热反应前后溶液 $\mathrm{pH}$ 值的变化可由下文 的相关反应方程进行解释)。

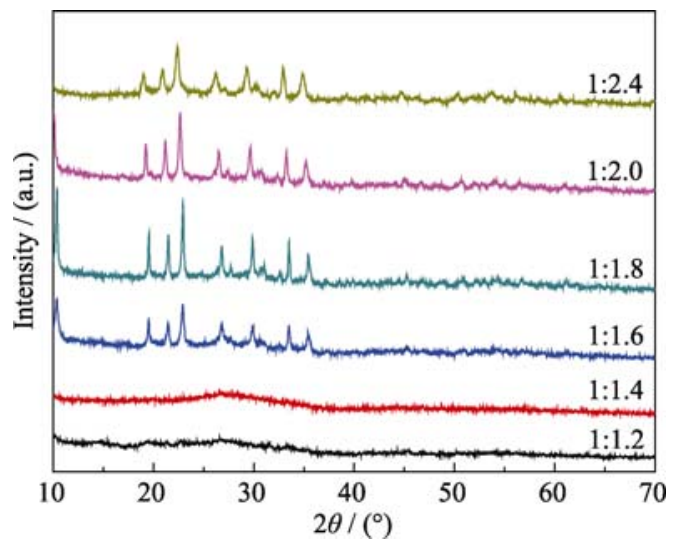

图 1 不同摩尔配比下所得产物的 XRD 图谱

Fig. 1 XRD patterns of products obtained at different molar ratio of raw materials
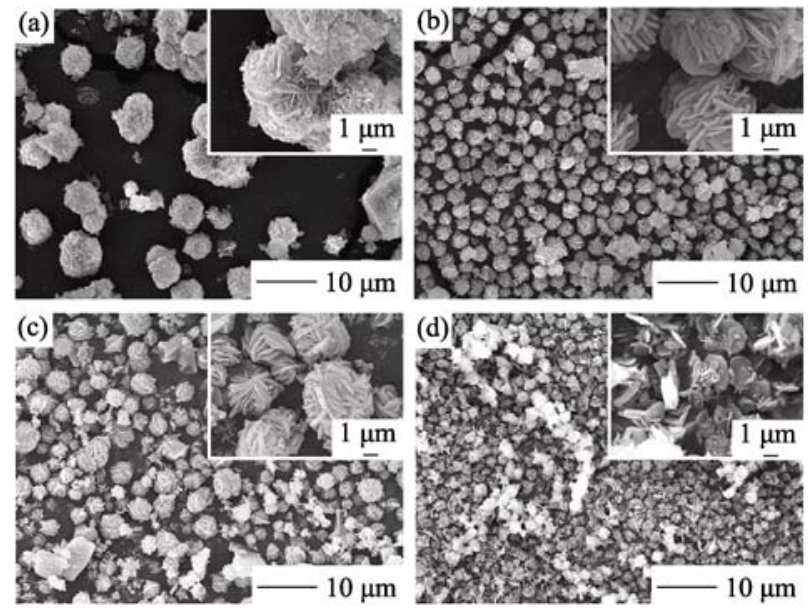

图 2 不同摩尔配比下所得产物的 SEM 照片

Fig. 2 SEM images of products obtained at different molar ratios of raw materials

(a) $1: 2.4$; (b) $1: 2.0$; (c) 1:1.8; (d) 1:1.6. Insets are enlarged images of part area in related images 
表 1 不同摩尔配比下水热前后溶液的 pH 值

Table 1 pH value of the solution with different molar ratio of raw materials before and after hydrothermal treatment

\begin{tabular}{ccccccc}
\hline Molar ratio & $1: 2.4$ & $1: 2.0$ & $1: 1.8$ & $1: 1.6$ & $1: 1.4$ & $1: 1.2$ \\
\hline Initial pH value & 7.24 & 7.00 & 6.72 & 6.57 & 6.43 & 6.06 \\
Final pH value & 5.72 & 5.66 & 5.58 & 5.49 & 5.38 & 5.32 \\
\hline
\end{tabular}

为考察 $\mathrm{pH}$ 对产物的影响, 分别使用 $\mathrm{NaOH}$ 溶液 和盐酸将加入 $\mathrm{ZrOCl}_{2} \cdot 8 \mathrm{H}_{2} \mathrm{O}$ 和 $\mathrm{Na}_{5} \mathrm{P}_{3} \mathrm{O}_{10}$ 的摩尔比为 1:2.0 的初配溶液的 $\mathrm{pH}$ 值调变为 8.0 和 6.0 , 图 3 为 他们在 $140^{\circ} \mathrm{C}$ 下应 $12 \mathrm{~h}$ 所得产物的 SEM 照片。由 图 3 和图 2(b)对比可以看出, 当 $\mathrm{pH}$ 改变时, 均会阻 止线团结构的形成而转变为无规则的颗粒, 而在不 调变的中性条件下获得产物的形貌最为均一。根据 以上实验结果, 以下水热实验所用原料均采用 $\mathrm{ZrOCl}_{2} \cdot 8 \mathrm{H}_{2} \mathrm{O}$ 和 $\mathrm{Na}_{5} \mathrm{P}_{3} \mathrm{O}_{10}$ 的摩尔比为 $1: 2.0$ 的比例进 行配置, 并且无需调变其 $\mathrm{pH}$ 值。

\section{2 水热温度的影响}

图 4 给出了不同温度下水热处理 $8 \mathrm{~h}$ 获得产物 的 XRD 图谱。由图可知, 相同水热时间, 当水热温 度在 $100 、 120$ 和 $140^{\circ} \mathrm{C}$ 时, 反应制得产物的 XRD 图谱没有明显的衍射峰, 说明产物为无定型结

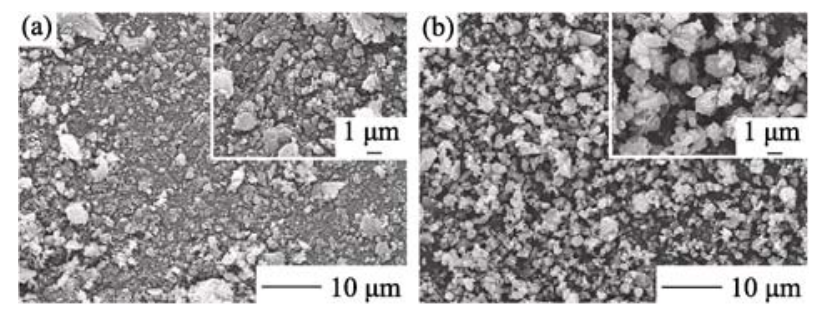

图 3 不同 $\mathrm{pH}$ 值下所得产物的 SEM 照片

Fig. 3 SEM images of products obtained at different $\mathrm{pH}$ values

(a) $\mathrm{pH}=8.0$; (b) $\mathrm{pH}=6.0$. Insets are enlarged images of part area in related images

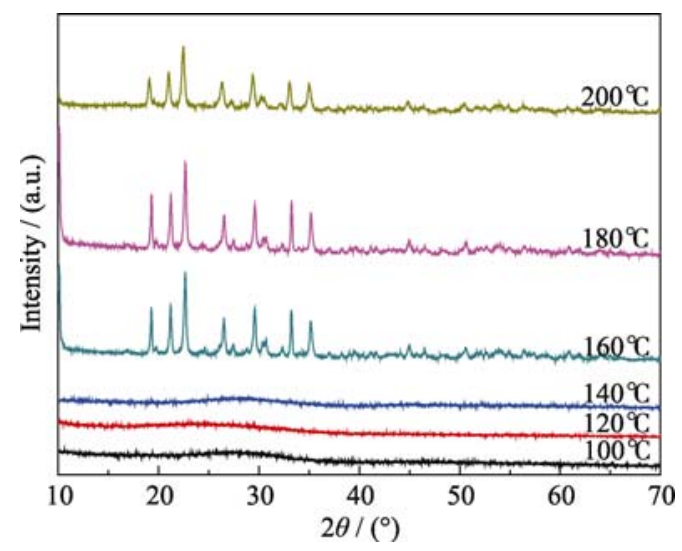

图 4 不同水热温度下所得产物的 XRD 图谱

Fig. 4 XRD patterns of products obtained at different temperatures
构。当水热温度为 $160 、 180$ 和 $200^{\circ} \mathrm{C}$ 时，反应产物 有相似的 XRD 图谱, 经分析晶相结构均为 $\mathrm{Na}_{2} \mathrm{Zr}$ $\left(\mathrm{PO}_{4}\right)_{2}$ 。另外从图 4 中还可以看到, 相比 $160^{\circ} \mathrm{C}$ 和 $200^{\circ} \mathrm{C}$ 时的产物衍射峰, 水热温度为 $180^{\circ} \mathrm{C}$ 时, 获得 产物的衍射峰强度最高, 峰形也最窄, 表明此温度 下获得的产物晶相发育更为良好。

图 5 给出了不同水热处理温度下水热 $8 \mathrm{~h}$ 获得 产物的 SEM 照片。由图 5 可以看出, 水热温度为 $100^{\circ} \mathrm{C}$ 时, 产物形貌为不规则的松散块状颗粒 (见图 5(a)); 升高水热温度，原来松散的块状颗粒向致密 的不规则块体转变(图 $5(\mathrm{~b}, \mathrm{c})$ ); 继续升高水热温度 到 $160 、 180 、 200^{\circ} \mathrm{C}$ 时，产物变为六角插片组装的 类线团状形貌，分散性好，大小均匀，颗粒尺寸在 $3 \mu \mathrm{m}$ 左右(图 5(d) (f)), 图 5(e)还显示了少量六角形 片状颗粒。

\section{3 水热时间的影响}

图 6 显示 $140^{\circ} \mathrm{C}$ 水热处理 $4 、 6$ 和 $8 \mathrm{~h}$ 时, 获得 的产物呈无定形形态。当水热处理时间为 $10 、 12$
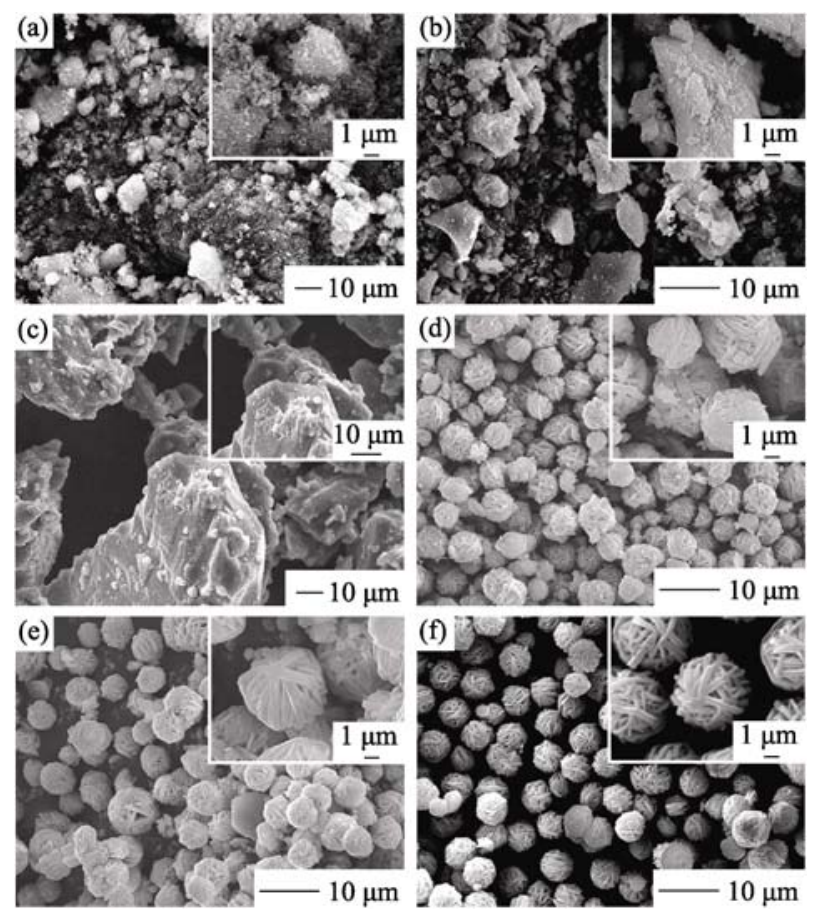

图 5 不同水热处理温度下获得产物的 SEM 照片

Fig. 5 SEM images of products obtained at different hydrothermal temperatures

(a) $100^{\circ} \mathrm{C}$; (b) $120^{\circ} \mathrm{C}$; (c) $140^{\circ} \mathrm{C}$; (d) $160^{\circ} \mathrm{C}$; (e) $180^{\circ} \mathrm{C}$; (f) $200^{\circ} \mathrm{C}$. Insets are enlarged images of part area in related images 


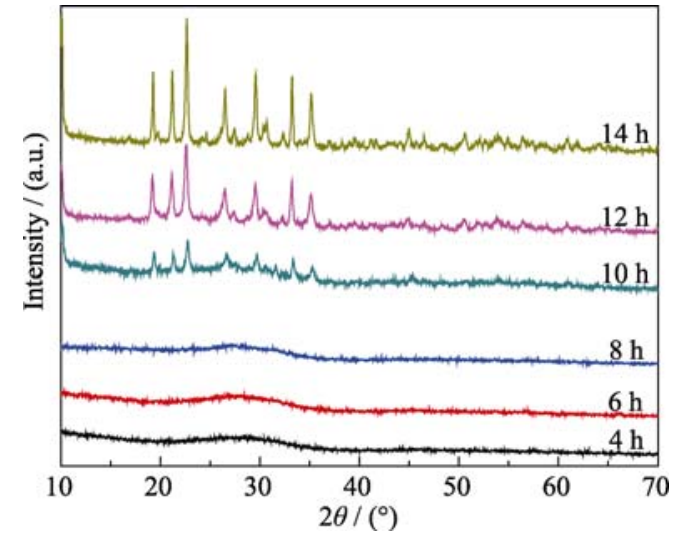

图 $6140{ }^{\circ} \mathrm{C}$ 下不同时间获得产物的 XRD 图谱

Fig. 6 XRD patterns of products obtained under different hydrothermal time at $140^{\circ} \mathrm{C}$

和 $14 \mathrm{~h}$ 时, 产物的 XRD 图谱类似，与标准图谱对 照可知, 产物晶相为 $\mathrm{Na}_{2} \mathrm{Zr}\left(\mathrm{PO}_{4}\right)_{2}$ 。随水热处理时 间的延长，产物的 XRD 图谱出现峰的位置没有发 生变化，说明延长水热处理时间不改变产物的相 结构，但峰强度逐渐增强，由谢乐公式计算可知， 产物晶粒尺寸也逐渐变大，这说明晶体逐渐发育 完整。

图 7 给出了水热温度 $140^{\circ} \mathrm{C}$ 不同水热处理时间 获得产物的 SEM 照片。从图 7 可以看出, 水热时 间为 $4 \mathrm{~h}$ 时，产物的微观形貌为散乱的不规则块体 (图 7(a)); 随着水热时间的延长, 块状物质逐渐聚 集长大并致密化(图 7(b,c)); 延长水热时间至 $10 \mathrm{~h}$ 时, 块状产物分化为颗粒大小较均匀的直径为 $3 \mu \mathrm{m}$ 左右的小球体，该小球体由表面粗粘且不规 整的插片状结构组装而成。同时有部分大块体未 分化完全，与形成的团聚在一起(图 7(d))。继续延 长水热时间，不规则块状产物逐渐消失，先前形 成的小球体转变为插片组装的类线团状产物，分 散性改善，显露出的组装片呈现为厚度均匀、表面 平滑的六角形状(见图 7(e, f))。将图 7 与图 5(c)对 照可知，温度越高，形成类线团状磷酸锆钠粉体 的时间越短。

\section{4 煅烧温度的影响}

图 8 为 $140^{\circ} \mathrm{C}$ 水热处理 $14 \mathrm{~h}$ 获得产物的 TG-DSC 曲线。图 8 中的 DSC 曲线存在一个明显吸热峰和一 个明显放热峰。第一个吸热峰在 $250^{\circ} \mathrm{C}$ 以前, 对照 $\mathrm{TG}$ 曲线可知，此阶段为失重阶段，此时的重量损失 达到 $8 \%$ 左右, 主要是样品中吸附水脱出引起。在 $550 \sim 850^{\circ} \mathrm{C}$ 时 $\mathrm{DSC}$ 曲线上出现放热峰, 对应的 $\mathrm{TG}$ 曲线没有明显重量损失，表明该峰的出现应为相转 变引起。
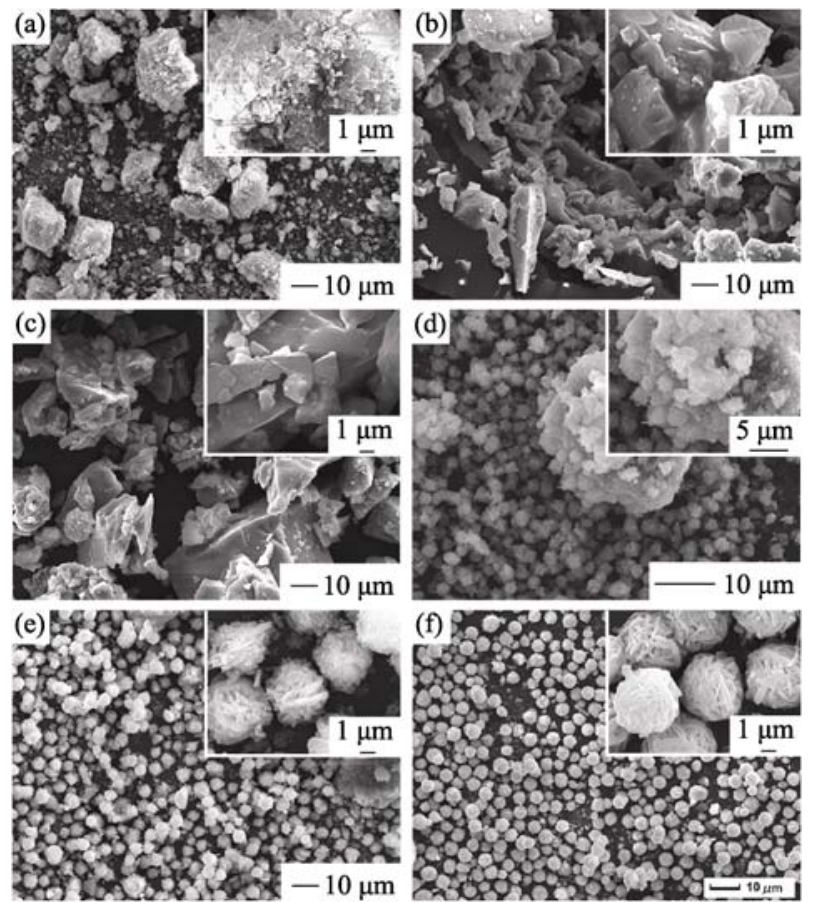

图 7 不同水热处理时间获得产物的 SEM 照片

Fig. 7 SEM images of products obtained under different hydrothermal time

(a) $4 \mathrm{~h}$; (b) $6 \mathrm{~h}$; (c) $8 \mathrm{~h}$; (d) $10 \mathrm{~h}$; (e) $12 \mathrm{~h}$; (f) $14 \mathrm{~h}$. Insets are enlarged images of part area in related images

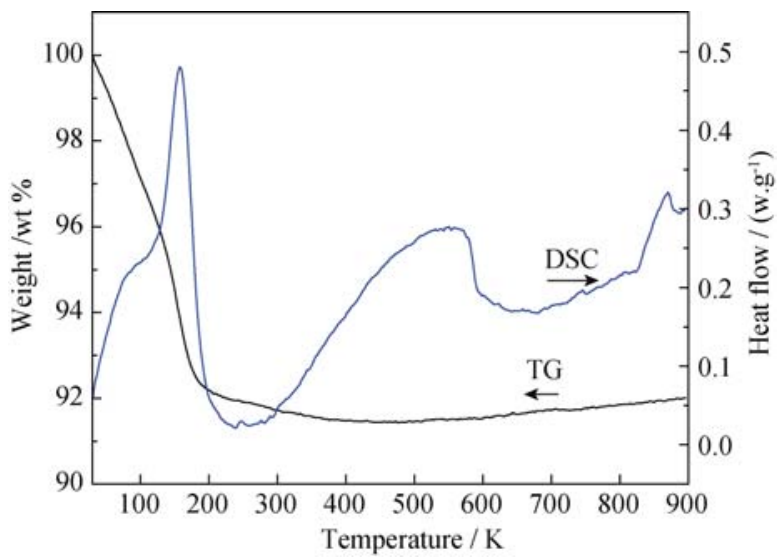

图 8 产物的 TG-DSC 曲线

Fig. 8 TG-DSC curve of the product

根据上述分析结果, 对该水热产物分别在 $600^{\circ} \mathrm{C}$ 和 $900^{\circ} \mathrm{C}$ 的温度下煅烧 $3 \mathrm{~h}$, 并对㷽烧后的粉体进行 XRD 检测和微观形貌观察。

图 9 为水热产物经 $600^{\circ} \mathrm{C}$ 和 $900^{\circ} \mathrm{C}$ 煅烧后的 $\mathrm{XRD}$ 图谱。由图 9(a)知, $600^{\circ} \mathrm{C}$ 煅烧后所得产物的主要晶 相由 $\mathrm{Na}_{2} \mathrm{Zr}\left(\mathrm{PO}_{4}\right)_{2}$ 和 $\mathrm{NaZr}_{2}\left(\mathrm{PO}_{4}\right)_{3}$ 两相组成。由图 9(b) 可知, $900^{\circ} \mathrm{C}$ 煅烧后, $\mathrm{NaZr}_{2}\left(\mathrm{PO}_{4}\right)_{3}$ 相的峰强较强, 成 为轱烧产物的主晶相，同时又有少量的 $\mathrm{Na}_{5} \mathrm{Zr}\left(\mathrm{PO}_{4}\right)_{3}$ 相生成。这说明 $\mathrm{Na}_{2} \mathrm{Zr}\left(\mathrm{PO}_{4}\right)_{2}$ 相会在高温下分解，形 成 $\mathrm{NaZr}_{2}\left(\mathrm{PO}_{4}\right)_{3}$ 相和 $\mathrm{Na}_{5} \mathrm{Zr}\left(\mathrm{PO}_{4}\right)_{3}$ 相。 

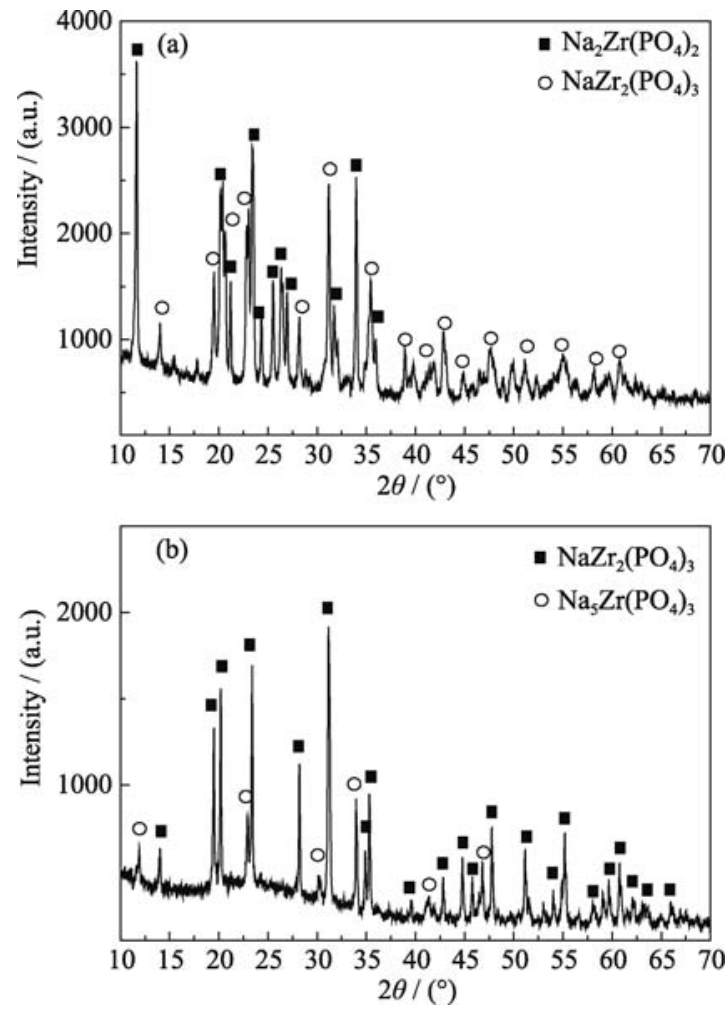

图 9 不同温度煅烧后产物的 XRD 图谱

Fig. 9 XRD patterns of the products calcined at $600^{\circ} \mathrm{C}$ (a) and $900^{\circ} \mathrm{C}(\mathrm{b})$

图 10 是水热产物炦烧后的 SEM 照片。由图 10 可以看到，经 $600^{\circ} \mathrm{C}$ 煅烧后类线团结构开始被破坏， 每个颗粒虽大致仍为插片组装的类线团结构, 但组 装片分布散乱, 表面较粗粮(图 10(a)); 经 $900^{\circ} \mathrm{C}$ 炦 烧后，类线团结构被彻底破坏，形成的颗粒大小不 一, 且有立方块状颗粒出现(图 10(b)), 这种立方颗 粒形貌与文献[15-16]报道类似。结合 XRD 分析可 知, 这种微观形貌的改变是由于高温下产物相结构 发生转变引起的。

\section{5 机理分析}

结合文献报道和本研究得到的实验结果, 水热 法生成类线团结构磷酸锆钠颗粒的可能机理如下:

八水合氧氯化锆水解呈酸性, 多聚磷酸钠主要 以三聚磷酸钠为主, 其水解呈碱性, 在溶液中他们

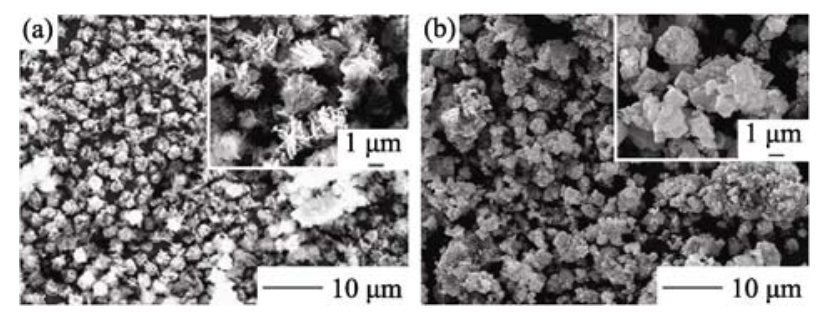

图 10 煅烧后产物的微观形貌

Fig. 10 Morphologies of the products calcined at different $600^{\circ} \mathrm{C}$ (a) and $900^{\circ} \mathrm{C}(\mathrm{b})$

Insets are enlarged images of part area in related images
的水解反应将相互促进，当他们的摩尔比为 $1: 2$ 时, 由于水解产物的水解电离达到平衡而使初配溶液呈 中性(见表 1), 相关反应方程式如下 ${ }^{[1,17]}$ :

$$
\begin{gathered}
\left(\mathrm{P}_{3} \mathrm{O}_{10}\right)^{5-}+4 \mathrm{H}_{2} \mathrm{O} \rightarrow 3\left(\mathrm{H}_{2} \mathrm{PO}_{4}\right)^{-}+2 \mathrm{OH}^{-} \\
(\mathrm{ZrO})^{2+}+4 \mathrm{OH}^{-}+\mathrm{H}_{2} \mathrm{O} \rightarrow\left[\mathrm{Zr}(\mathrm{OH})_{6}\right]^{2-} \\
{\left[\mathrm{Zr}(\mathrm{OH})_{6}\right]^{2-}+6\left(\mathrm{H}_{2} \mathrm{PO}_{4}\right)^{-} \rightarrow} \\
{\left[\mathrm{Zr}\left(\mathrm{HPO}_{4}\right)_{6}\right]^{8-}+6 \mathrm{H}_{2} \mathrm{O}} \\
{\left[\mathrm{Zr}\left(\mathrm{HPO}_{4}\right)_{6}\right]^{8-}+6 \mathrm{Na}^{+} \rightarrow\left[\mathrm{Zr}\left(\mathrm{NaPO}_{4}\right)_{6}\right]^{8-}+6 \mathrm{H}^{+}}
\end{gathered}
$$

水热反应过程中由于 $\mathrm{Na}^{+}$的置换效应(反应(3), (4)) 不断置换出 $\mathrm{H}^{+}$，而使反应后的溶液呈酸性(表 1$)$ 。

水热反应初期, 由于水解反应快速进行 (反应 (1)和(2)), 生成的大量 $\left[\mathrm{Zr}(\mathrm{OH})_{6}\right]^{2-}$ 将聚集为凝胶, 使此时得到的样品呈现为不规则块状(图 5(a),7(a)); 而反应(3)和反应(4)进行的速度相对缓慢，生成的 $\left[\mathrm{Zr}\left(\mathrm{NaPO}_{4}\right)_{6}\right]^{8-}$ 配位体(简称 $\mathrm{ZOP}$, 这种配位体结构 为中心的 $\mathrm{Zr}-\mathrm{O}_{6}$ 八面体与 $\mathrm{P}-\mathrm{O}_{4}$ 四面体通过 $\mathrm{O}$ 原子 相互连接, $\mathrm{P}-\mathrm{O}_{4}$ 四面体再与 $\mathrm{Na}-\mathrm{O}_{6}$ 八面体相互连接 而成 $)^{[1,6]}$ 作为生长基元将相互取向连接 ${ }^{[14,18]}$ 形成微 小晶粒。温度的适当升高, ZOP 和微小晶粒形成的 速度将会变快，这些微小晶粒包裹在 $\left[\mathrm{Zr}(\mathrm{OH})_{6}\right]^{2-}$ 凝 胶中使得到的产物不断致密化，形成致密的无规则 块体(图 5(a) (c)和图 7(a) (c)); 当微小晶粒生长到 一定规模时，许多这样的晶粒因相互靠近会再次取 向连接形成更大的晶体，但此时胶体状物质未分化 完全, 会与晶体表面发生粘连, 使产物的形貌由致 密的无规则块体变为表面粗糙的插片组装而成的球 状颗粒(图 7(c)、(d)); 在晶体生长过程中, 由于原料 的消耗，溶液中 ZOP 浓度将不断降低而使其取向连 接的各向异性增大，从而使晶体在某些方向相对其 他方向生长较快，最终形成的颗粒为类线团状，暴 露的组装片由开始的不规整状生长为一定厚度的平 滑六角片状 ${ }^{[11,14]}$ (图 7(d) (f)), 晶粒尺寸增大, 晶体 发育得更加完整(图 6), 另外, 少量后期才形成的微 小晶粒由于各向异性生长，长大后表现为少量六角 片状颗粒的出现(图 5(e))。

\section{3 结论}

本研究采用水热法, 首次制备出六角形插片组 装而成的类线团形貌的 $\mathrm{Na}_{2} \mathrm{Zr}\left(\mathrm{PO}_{4}\right)_{2}$ 粉体。研究发现, 当原料摩尔比为 $n\left(\mathrm{ZrOCl}_{2} \cdot 8 \mathrm{H}_{2} \mathrm{O}\right): n\left(\mathrm{Na}_{5} \mathrm{P}_{3} \mathrm{O}_{10}\right)=1: 2$ 时, 初配溶液的 $\mathrm{pH}$ 值恰为中性, 将此溶液在 $140^{\circ} \mathrm{C}$ 下水热 $14 \mathrm{~h}$ 得到的产物为分散性较好, 尺寸较均一 的类线团状 $\mathrm{Na}_{2} \mathrm{Zr}\left(\mathrm{PO}_{4}\right)_{2}$ 粉体, 而改变此溶液的 $\mathrm{pH}$ 值会破坏类线团结构的形成; 采用以上原料配比, 
无需调变 $\mathrm{pH}$ 值, 在合适的温度及时间下均能生成 类线团状 $\mathrm{Na}_{2} \mathrm{Zr}\left(\mathrm{PO}_{4}\right)_{2}$ 粉体, 且 $180^{\circ} \mathrm{C}$ 时晶形发育最 好; 随水热时间的延长, 制得的产物形貌由不规则 块体向类线团形貌转变, 晶相结构由无定型结构向 $\mathrm{Na}_{2} \mathrm{Zr}\left(\mathrm{PO}_{4}\right)_{2}$ 晶相结构转变, 且温度越高, 形成这种 形貌所需水热时间越短。这种类线团结构的形成是 由于 $\mathrm{ZOP}$ 生长基元取向连接的各向异性造成的。在 $600^{\circ} \mathrm{C}$ 以上高温門烧过程中这种结构会被破坏, 单 相的 $\mathrm{Na}_{2} \mathrm{Zr}\left(\mathrm{PO}_{4}\right)_{2}$ 将会分解, 形成 $\mathrm{NaZr}_{2}\left(\mathrm{PO}_{4}\right)_{3}$ 主晶相 和少量的 $\mathrm{Na}_{5} \mathrm{Zr}\left(\mathrm{PO}_{4}\right)_{3}$ 相。

\section{参考文献:}

[1] PET'KOV V I. Complex phosphates formed by metal cations in oxidation states I and IV. Russian Chemical Reviews, 2012, 81(1): 606-637.

[2] ZHANG BIAO, GUO JING-KUN, HUANG XIAO-XIAN, et al. Properties of [NZP] structure-functional materials. Journal of Inorganic Materials, 1996, 11(1): 14-19.

[3] SCHEETZ B E, AGRAWAL D K, BREVAL E, et al. Sodium zirconium phosphate(NZP) as a host structure for nuclear waste immobilization: a review. Waste Management, 1994, 14(6): 489-505.

[4] BURDA C, CHEN X, NARAYANAN R, et al. Chemistry and properties of nanocrystals of different shapes. Chemical Reviews, 2005, 105(4): 1025-1102.

[5] ZHANG Q, LIU S J, YU S H. Recent advances in oriented attachment growth and synthesis of functional materials: concept, evidence mechanism, and future. Journal of Material Chemistry, 2009, 19(1): 191-207.

[6] PET'KOV V I, ASABINA E A. Complex phosphates, containing elements with oxidation degree +1 and +4 . Physics Procedia, 2013, 44: $166-176$.

[7] ZHU LIN-HUA, LIAO XUE-PIN, LANG XIAO-CHUAN, et al. Synthesis of $\mathrm{Ca}_{1-x} \mathrm{Ba}_{x} \mathrm{Zr}_{4}\left(\mathrm{PO}_{4}\right)_{6}$ phosphate ceramic and its thermal shock resistance performance of near-zero thermal expansion composition. Journal of Inorganic Materials, 2001, 16(3): 452-458.

[8] YIN Z, SAKAMOTO Y, YU J, et al. Microemulsion based synthesis of titanium phosphate nanotubes via amine extraction system. Journal of the American Chemical Society, 2004, 126(29):
8882-8883.

[9] LI Y H, LING Y H, BAI X D. Preparation and characterization of anisotropic ammonium titanium phosphate crystals via hydrothermal route. Key Engineering Materials, 2005, 280-283: 597-600.

[10] PAVLOVA S N, SADYKOV V A, ZABOLOTNAYA G V, et al. The influence of solid precursors nature on structural, textural and surface properties of framework zirconium phosphates synthesized via mechanochemical activation. Solid State Ionics, 2001, 141-142: 683-688.

[11] ZHANG XUE-HUA, LUO HAO-SU, ZHONG WEI-ZHUO. Anionic corrdination polyhedron growth units mode and its application in crystal growth. Science in China Series E, 2004, 34(3): 241-253.

[12] PENN R L, BANFIELD J F. Morphology development and crystal growth in nanocrystalline aggregates under hydrothermal conditions: insights from titania. Geochimica et Cosmochimica Acta, 1999, 63(10): 1549-1557.

[13] ECHEVERRÍA J, ALVAREZ S. Application of symmetry operation measures in structure inorganic chemistry. Inorganic Chemistry, 2008, 47(23): 10965-10970.

[14] NAI J, WU J, GUO L, et al. Coordination polyhedra: a probable basic growth unit in solution for the crystal growth of inorganic nonmetallic nanomaterials? Crystal Growth Design, 2012, 12(5): 2653-2661.

[15] AMBARISH D, AMIT D G, DEBRATA B, et al. A comparative study of conventionally sintered, microwave sintered and hot isostatic press sintered NZP and CZP structures interacted with fluoride. Ceramics International, 2013, 39(8): 9351-9359.

[16] ORDÓÑEZ-REGIL E, CONTRERAS-RAMÍREZ A, FERNÁNDEZVALVERDE S M, et al. Crystal growth and thermoluminescence response of $\mathrm{NaZr}_{2}\left(\mathrm{PO}_{4}\right)_{3}$ at high gamma radiation doses. Journal of Nuclear Materials, 2013, 443(1/2/3): 417-423.

[17] GEORGE A, SEENA P T. Thermal studies on zirconium hydroxide gel formed by aqueous gelation. Journal of Thermal Analysis and Calorimetry, 2012, 110(3): 1037-1041.

[18] BANFIELD J F, WELCH S A, ZHANG H, et al. Aggregation-based crystal growth and microstructure development in natural iron oxyhydroxide biomineralization products. Science, 2000, 289(5480): 751-754. 